\title{
BMJ Open Agreement between capillary and venous lactate in emergency department patients: prospective observational study
}

\author{
Colin A Graham, ${ }^{\oplus 1,2}$ Ling Yan Leung, ${ }^{\oplus 1}$ Ronson SL Lo, ${ }^{1}$ Kwok Hung Lee, ${ }^{1,2}$ \\ Chun Yu Yeung, ${ }^{1}$ Suet Yi Chan, ${ }^{1}$ Giles N Cattermole, ${ }^{1}$ Kevin KC Hung ${ }^{\circ 1,2}$
}

To cite: Graham CA, Leung LY, Lo RSL, et al. Agreement between capillary and venous lactate in emergency department patients: prospective observational study. BMJ Open 2019;9:e026109. doi:10.1136/ bmjopen-2018-026109

- Prepublication history for this paper is available online. To view these files, please visit the journal online (http://dx.doi. org/10.1136/bmjopen-2018026109).

Received 17 August 2018 Revised 4 January 2019 Accepted 22 February 2019

Check for updates

(C) Author(s) (or their employer(s)) 2019. Re-use permitted under CC BY-NC. No commercial re-use. See rights and permissions. Published by BMJ.

${ }^{1}$ Accident and Emergency Medicine Academic Unit, Chinese University of Hong Kong, Shatin, Hong Kong

${ }^{2}$ Emergency Department, Prince of Wales Hospital, Shatin, Hong Kong SAR

Correspondence to

Dr Kevin KC Hung;

kevin.hung@cuhk.edu.hk

\section{ABSTRACT}

Objectives Capillary blood lactate testing with handheld analysers has great advantages to reduce the time needed for clinical decisions, and for extended use in the prehospital setting. We investigated the agreement of capillary lactate measured using handheld analysers (CL-Nova and CL-Scout+ measured by Nova and Lactate Scout+ analyzers) and the reference venous level assessed using a point-of-care testing (POCT) blood gas analyser (VL-Ref).

Design A prospective observational study.

Setting A university teaching hospital emergency department in Hong Kong.

Participants Patients triaged as 'urgent' (Category 3 of a 5-point scale), aged $\geq 18$ years during 2016 were eligible. 240 patients (mean age 69.9 years) were recruited. Primary and secondary outcome measures The primary outcome measure was the agreement of the capillary blood lactate level measured by handheld lactate analyser when compared with the reference standard technique, namely venous blood samples obtained by venepuncture and analysed using the blood gas analyser. The secondary outcome measure was the difference in values of venous lactate using blood gas analysers and handheld lactate analysers.

Results The results of VL-Ref ranged from 0.70 to $5.38 \mathrm{mmol} / \mathrm{L}$ (mean of $1.96 \mathrm{mmol} / \mathrm{L}$ ). Regarding capillary lactate measurements, the bias (mean difference) between VL-Ref and CL-Scout+ was -0.22 with $95 \%$ limits of agreement (LOA) of -2.17 to $1.73 \mathrm{mmol} / \mathrm{L}$ and the bias between VL-Ref and CL-Nova was 0.46 , with LOA of -1.08 to $2.00 \mathrm{mmol} / \mathrm{L}$. For venous lactate, results showed the bias between VL-Ref and VL-Scout+ was 0.22 with LOA being -0.46 to $0.90 \mathrm{mmol} / \mathrm{L}$, and the bias between VL-Ref and VL-Nova was $0.83 \mathrm{mmol} / \mathrm{L}$ with $\mathrm{LOA}-0.01$ to $1.66 \mathrm{mmol} / \mathrm{L}$.

Conclusion Our study shows poor agreement between capillary lactate and reference values. The study does not support the clinical utility of capillary lactate POCT. However, venous lactate measured by Scout+ handheld analyser may have potential for screening patients who may need further testing.

Trial registration number NCT02694887.

\section{INTRODUCTION}

Elevated lactate levels are a common finding in acutely unwell patients. Blood lactate level

\section{Strengths and limitations of this study}

- This study provided important insights into the accuracy of point-of-care testing (POCT) capillary and venous blood lactate in undifferentiated Hong Kong adult patients with urgent conditions.

- We used two handheld devices to determine the accuracy of the POCT capillary and venous lactate.

- Bland-Altman agreement analyses of the mean difference in lactate between the reference standard and the measurements using handheld analysers were performed.

- However, the overall reliability of the procedure may be affected by the design of the analysers and endogenous patient factors including oxygen levels, skin temperature and altered haematocrit levels.

is a good predictor of patient outcome and high lactate levels are associated with high morbidity and mortality. ${ }^{12}$ Lactate may be detected and measured in all blood samples: arterial, venous and capillary. Good correlation of lactate concentrations between arterial and venous blood samples have been noted. ${ }^{34}$ Due to the possible risk and the additional time required to obtain an arterial sample, it has been suggested that venous blood gas (VBG) analysis can replace arterial blood gas analysis in the emergency department (ED).$^{5}$ In particular, venous lactate has demonstrated to be reliable in the management of trauma and sepsis in the ED. ${ }^{67}$

Currently, blood lactate can be measured formally in the laboratory, using blood gas analysers or with handheld devices. Strong correlations have been shown between blood lactate levels in samples analysed in the central laboratory, by blood gas analysers ${ }^{8}$ and handheld devices. ${ }^{1011}$ The turnover time of using handheld devices is significantly shorter than the samples analysed in the central laboratory. ${ }^{10}$ Comparing to arterial and venous blood sample, obtaining capillary blood samples is quick, less painful ${ }^{12}$ and the technique is already well known to ED 
healthcare workers. Along with its small blood volume requirement for analysis and rapid availability of results, handheld analysers could potentially allow earlier identification of patients with a higher risk of mortality. Handheld analysers could reduce the time needed for clinical decisions and potentially improve the quality of care, with the potential to extend their use to the triage area or the prehospital setting.

However, there are conflicting reports on the accuracy of this rapid capillary lactate measurement using handheld devices. Previous studies have inconsistent results on agreement between capillary blood lactate and venous blood lactate. Capillary blood lactate has been shown to have good agreement with lactate levels in venous samples in newborns. ${ }^{13}$ In contrast, poor agreement between capillary lactate and venous blood lactate were observed in adult ED patients. ${ }^{14-16}$ The small sample size ranging from 24 to 120 of previous studies limited the generalisation of these studies.

Crowding in ED prolongs patient waiting times, especially from triage to physician assessment. Point-of-care testing (POCT) such as rapid lactate measurement could aid early recognition of patients at high risk of mortality and morbidity. ${ }^{617}$ The aim of this study was to study the agreement of handheld lactate analysers for the measurement of capillary lactate as compared with a reference standard, namely venous blood lactate level assessed using a blood gas analyser.

We hypothesised that there would be good agreement, as defined by Bland-Altman analysis, ${ }^{18}$ between capillary blood lactate levels analysed with handheld analysers compared with venous blood lactate levels analysed with a blood gas analyser. There is no standard definition of what is considered good agreement. We considered that if the point estimate of the limit of agreement $(\mathrm{LOA})^{18}$ was within $\pm 0.4 \mathrm{mmol} / \mathrm{L}$ (equivalent to $\pm 20 \%$ of the upper limit of normal lactate value of $2 \mathrm{mmol} / \mathrm{L}$ ) from the point estimate of the bias would be clinically acceptable. Similarly, good agreement was defined as having LOA within $\pm 0.2 \mathrm{mmol} / \mathrm{L}$ (equivalent to $\pm 10 \%$ of upper limit of the normal lactate value of $2 \mathrm{mmol} / \mathrm{L}$ ) from the point estimate of the bias.

\section{MATERIAL AND METHODS \\ Study design and patients}

This was a prospective observational study of patients presenting to the ED of Prince of Wales Hospital, a tertiary university teaching hospital in Hong Kong with an annual census of 140000 attendances.

Inclusion criteria were patients aged $\geq 18$ years presenting to the ED who were triaged as category 3 (urgent). ${ }^{19}$ Exclusion criteria included pregnant patients or those aged $\leq 17$ years.

\section{Sample collection and lactate analysis}

Venous and capillary blood samples were collected for lactate analysis. Venous blood samples (approximate
$1 \mathrm{~mL}$ ) were collected by venepuncture while capillary blood samples were collected by finger-prick with a disposable lancet. Venous lactate levels measured by blood gas analyser (Siemens Automatic QC RAPID Systems RAPID Point@500, Siemens Healthcare Diagnostics, New York, USA) served as the reference standard. Capillary lactate levels were immediately analysed by two handheld lactate analysers: Nova StatStrip Xpress Lactate Meter (Nova Biomedical, Waltham, Massachusetts, USA) and Lactate Scout+ (EKF Diagnostics, Leipzig, Germany). Venous lactate levels were also measured on the two handheld lactate analysers. All lactate measurements were performed within 15 min after obtaining the venous sample.

\section{Data collection}

Basic patient characteristics including age and sex, vital parameters recorded at triage (blood pressure, heart rate, respiratory rate and temperature) were also documented.

\section{Outcomes}

The primary outcome measure was the agreement of the capillary blood lactate level measured by handheld lactate analyser when compared with the reference standard technique, namely venous blood sample obtained by venepuncture and analysed using the blood gas analyser.

The secondary outcome measure was the difference in values of venous lactate using blood gas analyser and handheld lactate analysers.

\section{Statistical analysis}

Agreement of handheld lactate analysers with blood gas analyser was analysed by using Bland-Altman agreement analysis. ${ }^{18} 95 \%$ (mean $\pm 1.96 \mathrm{SD}$ ) LOA were generated for each Bland-Altman analysis. All analyses were carried out using MedCalc V.15.8 for Windows (Ostend, Belgium).

\section{Sample size}

The sample size suggested based on the Bland-Altman method was $200 .{ }^{1820}$ We allowed an extra $20 \%$ for unforeseen circumstances, and therefore the final sample size for this study was $240(200 \times 120 \%=240)$.

\section{DEFINITIONS}

VL-Ref, the venous lactate level measured by blood gas analyser, served as the reference standard. VL-Nova and VL-Scout+ arevenous lactate levels measured by handheld lactate analysers Nova StatStrip Xpress Lactate Meter and Lactate Scout+ Analyzer, respectively. CL-Nova and CL-Scout+ arecapillary lactate levels measured by handheld lactate analysers Nova StatStrip Xpress Lactate Meter and Lactate Scout+ Analyzer, respectively.

\section{PATIENT AND PUBLIC INVOLVEMENT}

The development of research question and outcome measures were not informed by patient's priorities, experience and preferences. Patients were not involved in the 
Table 1 Basic characteristics of 240 patients

\begin{tabular}{lc}
\hline & Value \\
\cline { 2 - 2 } Characteristics & Mean (range) \\
\hline Age & $69.9 \pm 16.1(20-99)$ \\
Sex (male) & $130(54.2 \%)$ \\
\hline Temperature $\left({ }^{\circ} \mathrm{C}\right)$ & $36.9 \pm 0.91(34.9-40.4)$ \\
\hline Systolic blood pressure $(\mathrm{mm} \mathrm{Hg})$ & $141.7 \pm 23.7(93-217)$ \\
Diastolic blood pressure $(\mathrm{mm} \mathrm{Hg})$ & $74.8 \pm 15.5(31-143)$ \\
Heart rate (/min) & $83.5 \pm 18.9(45-132)$ \\
Respiratory rate $(/ \mathrm{min})$ & $17.1 \pm 4.1(12-32)$ \\
\hline Lactate measurement & \\
Venous (Reference) & $2.0 \pm 0.9(0.7-5.4)$ \\
Venous (Nova) & $1.1 \pm 0.6(0.3-4.7)$ \\
Venous (Scout+) & $1.7 \pm 0.8(0.5-5.5)$ \\
\hline Capillary (Nova) & $1.5 \pm 0.8(0.4-6.0)$ \\
\hline Capillary (Scout+) & $2.2 \pm 1.2(0.6-9.2)$ \\
\hline
\end{tabular}

All continuous data are expressed as mean \pm SD and range.

design of studies in Hong Kong at the time this study was proposed. They were not involved in the recruitment or the conduct of the study. Results have been made available for patients on request.

\section{RESULTS}

Two hundred and forty patients (mean age 69.9 years; $54.2 \%$ males) were recruited (table 1 ). Of the 240 patients, $63.8 \%$ showed a venous lactate level (VL-Ref) $<2 \mathrm{mmol} / \mathrm{L}$ and $36.2 \% \geq 2 \mathrm{mmol} / \mathrm{L}$. VL-Ref ranged from 0.70 to $5.38 \mathrm{mmol} / \mathrm{L}$, mean $1.96 \mathrm{mmol} / \mathrm{L}$ (SD $0.88 \mathrm{mmol} / \mathrm{L})$.

Out of the samples from the 240 subjects, two CL-Scout +samples and two CL-Nova samples were lost due to analyser errors and subsequent refusals from patients to have blood samples redrawn. Samples of VL-Ref, VL-Scout+ and VL-Nova for data analysis were 240 collectively.

Mean differences and (Bland-Altman) LOA, together with $95 \%$ CIs, for differences between capillary and venous readings from the two handheld devices and the reference, blood gas, values are given in table 2 , and separately for reference values $<2 \mathrm{mmol} / \mathrm{L}$ and $\geq 2 \mathrm{mmol} / \mathrm{L}$ in table 3. Bland-Altman plots are shown in figure 1.

\section{DISCUSSION}

This study demonstrates that there is low systematic bias but a wide LOA between the capillary lactate measured with the Scout +analyser (CL-Scout+) and the 'reference standard' venous lactate measured by the blood gas analyser (VL-Ref). Furthermore, there is high systematic bias and a wide LOA between CL-Nova and VL-Ref. These findings suggest that capillary lactate measurement by handheld lactate analysers is not interchangeable with the current reference methods for lactate for diagnostic purposes.

In common with our study, a small mean difference but wide LOAs were observed between capillary lactate and reference methods in other ED patients ${ }^{14} 15$ and trauma patients. ${ }^{14}$ The sample size of these three studies ranged from 24 to 120 patients. Our current study with a larger sample size $(n=240)$ confirms the poor agreement between capillary lactate and venous lactate. These findings indicate that there is poor precision between the two methods. Such poor precision suggests that handheld capillary lactate testing cannot replace the current reference methods for lactate estimation in the ED. In contrast, good agreement between capillary and arterial lactate was observed in newborns in the neonatal ICU. ${ }^{1321}$ Further studies are required to compare the use of handheld lactate analysers between children and adult patients.

The present findings showed that different results were obtained using different handheld lactate analysers. The results suggest that the Scout +overestimates lactate levels and the Nova underestimates lactate levels. Overestimation of lactate levels may lead to unnecessary clinical intervention, while underestimation of lactate levels may delay intervention. ${ }^{22}$

Among venous lactate level measurements, both the Scout +and Nova lactate handheld analyser failed to meet the definitions for good/clinically acceptable agreements

Table 2 Agreement between capillary blood lactate and venous blood lactate

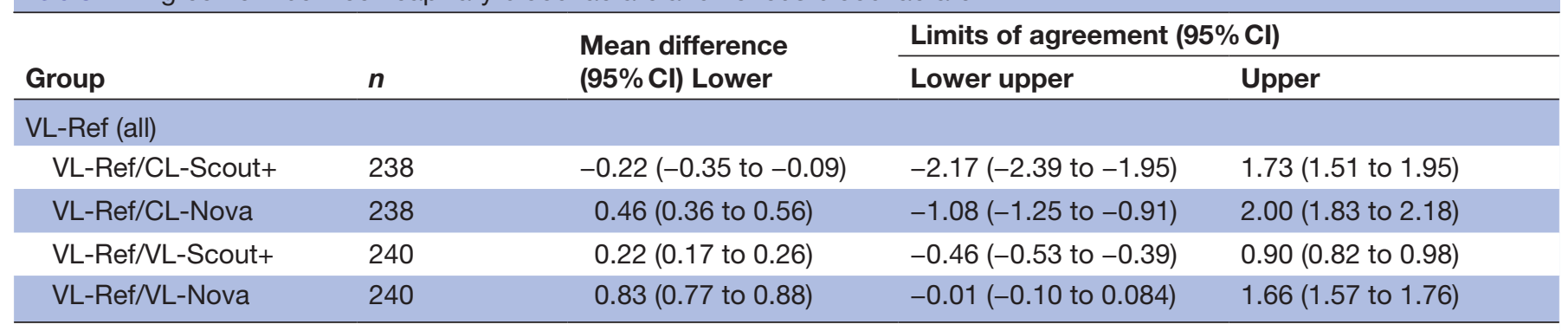

The point estimate of the level of agreement (LOA) being within $\pm 0.4 \mathrm{mmol} / \mathrm{L}$ from the point of the bias considered as clinically acceptable; and the point estimate of the LOA within $\pm 0.2 \mathrm{mmol} / \mathrm{L}$ from the point of the bias considered as good agreement. 
Table 3 Agreement between capillary blood lactate and venous blood lactate of subgroup VL-Ref $<2 \mathrm{mmol} / \mathrm{L}$ and VLRef $\geq 2 \mathrm{mmol} / \mathrm{L}$

\begin{tabular}{|c|c|c|c|c|}
\hline \multirow[b]{2}{*}{ Group } & \multirow[b]{2}{*}{$\mathbf{n}$} & \multirow{2}{*}{$\begin{array}{l}\text { Mean difference } \\
(95 \% \mathrm{Cl}) \text { Lower }\end{array}$} & \multicolumn{2}{|c|}{ Limits of agreement $(95 \% \mathrm{Cl})$} \\
\hline & & & Lower upper & Upper \\
\hline \multicolumn{5}{|l|}{ VL-Ref $<2 \mathrm{mmol} / \mathrm{L}$} \\
\hline VL-Ref/CL-Scout+ & 151 & $-0.32(-0.47$ to -0.17$)$ & $-2.13(-2.39$ to -1.88$)$ & $1.49(1.23$ to 1.74$)$ \\
\hline VL-Ref/CL-Nova & 149 & $0.20(0.10$ to 0.31$)$ & $-1.06(-1.24$ to -0.88$)$ & 1.46 (1.29 to 1.64$)$ \\
\hline VL-Ref/VL-Scout+ & 151 & $0.16(0.12$ to 0.20$)$ & $-0.36(-0.43$ to -0.28$)$ & 0.67 (0.60 to 0.75$)$ \\
\hline VL-Ref/VL-Nova & 151 & $0.62(0.59$ to 0.65$)$ & $0.23(0.18$ to 0.29$)$ & 1.00 (0.95 to 1.06$)$ \\
\hline \multicolumn{5}{|l|}{ VL-Ref $\geq 2 \mathrm{mmol} / \mathrm{L}$} \\
\hline VL-Ref/CL-Scout+ & 87 & $-0.04(-0.28$ to 0.19$)$ & $-2.18(-2.58$ to -1.78$)$ & 2.09 (1.70 to 2.49$)$ \\
\hline VL-Ref/CL-Nova & 89 & 0.89 (0.72 to 1.07$)$ & $-0.71(-1.01$ to -0.42$)$ & 2.50 (2.20 to 2.79 ) \\
\hline VL-Ref/VL-Scout+ & 89 & $0.32(0.22$ to 0.41$)$ & $-0.55(-0.71$ to -0.39$)$ & 1.18 (1.02 to 1.34$)$ \\
\hline VL-Ref/VL-Nova & 89 & 1.18 (1.08 to 1.28$)$ & 0.25 (0.077 to 0.42$)$ & 2.11 (1.94 to 2.29$)$ \\
\hline
\end{tabular}

The point estimate of the level of agreement (LOA) being within $\pm 0.4 \mathrm{mmol} / \mathrm{L}$ from the point of the bias considered as clinically acceptable; and the point estimate of the LOA within $\pm 0.2 \mathrm{mmol} / \mathrm{L}$ from the point of the bias considered as good agreement.

compared with the reference blood gas analyser (VL-Ref). This is in contrast with the good agreement found between venous blood lactate using a handheld lactate analyser (i-STAT) and a central laboratory lactate analysis $^{10}{ }^{11}$ in previous studies.

\section{A}
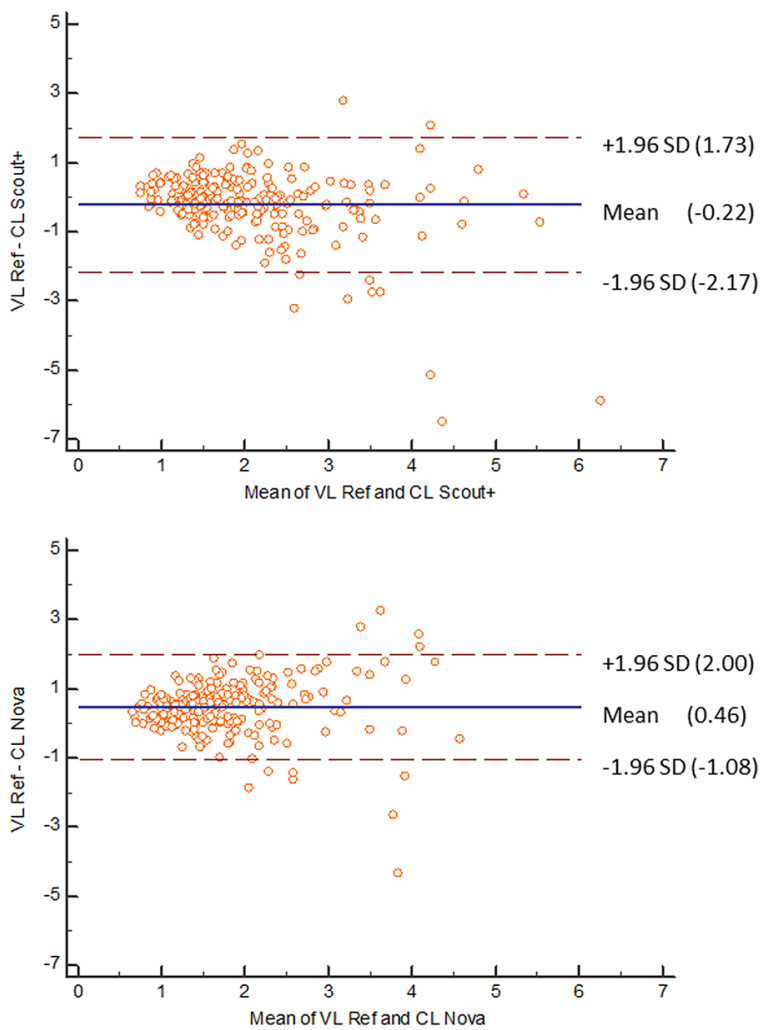

Although failing to meet the acceptable agreement criteria, better agreement was noted in the subgroup of patients with reference lactate levels $<2 \mathrm{mmol} / \mathrm{L}$ between VL-Scout+ and VL-Ref. This suggests that within normal lactate levels, the Scout+ handheld lactate

B
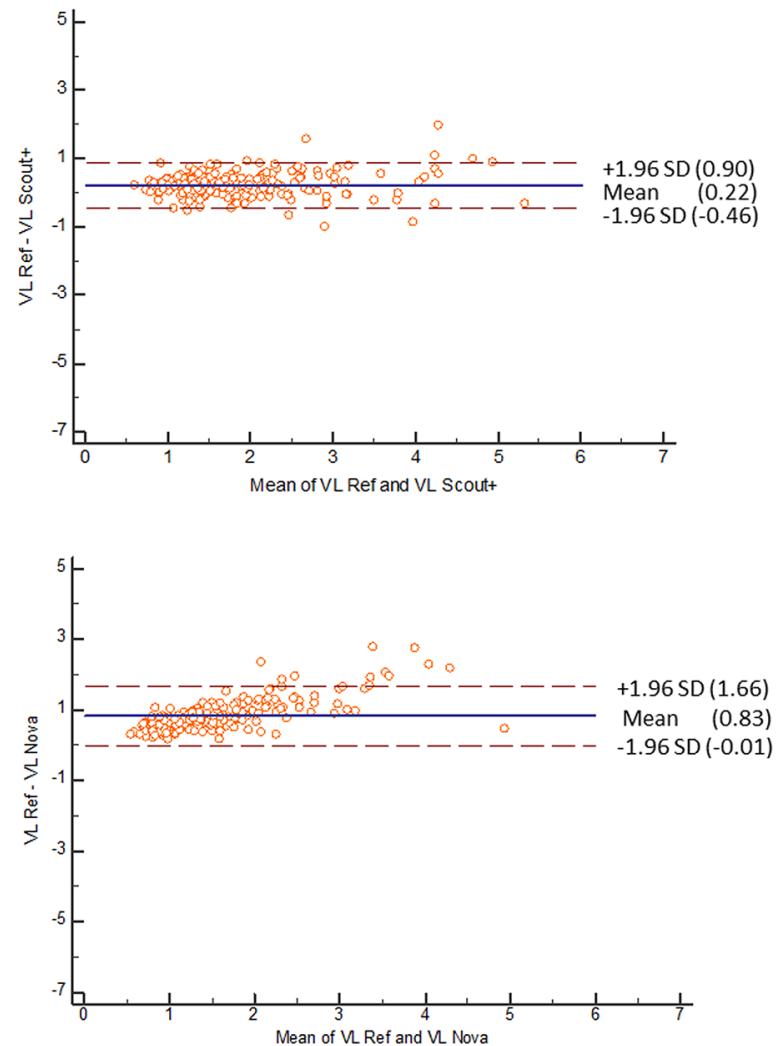

Figure 1 Bland-Altman plots showing the agreement between venous blood lactate using blood gas analyser (VL-Ref) and (A) capillary blood lactate level obtained by handheld analyser Scout+ (CL-Scout+) and Nova (CL-Nova), (B) venous blood lactate level obtained by handheld analyser Scout+ (VL-Scout+) and Nova (VL-Nova). Solid line represents bias; dashed lines represent upper and lower $95 \%$ limits of agreement. 
analyser may have the potential to screen patients who should or should not have further formal lactate measurements using VBG analysis as a POCT or in the central laboratory. Screening ED venous lactate levels by handheld analyser could provide information to shorten the time to identification of patients at risk, to allow rapid decision making for further treatment. However, the poor agreement between VL-Nova and VL-Ref suggests that the Nova lactate handheld analyser is not suitable for clinical use in the ED.

Our results do not currently support the use of capillary lactate measurement for diagnostic purposes. However, portable handheld analysers have the great advantage of reduced turn-around times, requiring only a small amount of blood, affordable cost, and being easily accessible $^{2324}$ allowing the use of this procedure in hospital, and in the prehospital setting including nursing homes. Further improvements in agreement are required to allow stronger recommendations for the routine use of handheld lactate analysers.

\section{Limitations of the study}

Capillary blood lactate testing with handheld analysers has great potential advantages. However, the overall reliability of the procedure may be affected by the design of the analysers, endogenous patient factors and operational errors. Endogenous patient factors such as oxygen levels, skin temperature and altered haematocrit may influence the results of handheld lactate analysers. ${ }^{25} 26$ Furthermore, operational errors may contribute towards the imprecision of the capillary lactate testing. Capillary lactate testing only requires a small amount of blood for analysis and this may allow easy contamination of the sample to occur, by sweat, ${ }^{25}$ disinfectant and alcohol swabs, for example.

\section{CONCLUSION}

The current study shows poor agreement between capillary lactate and the reference method. Our results do not support the clinical use of capillary lactate POCT. However, venous lactate measured by Scout+ handheld analyser may have potential for screening patients who may need further testing using more definitive methods.

Acknowledgements We would like to thank all the staff in the Emergency Department of the Prince of Wales Hospital of Hong Kong for their suggestions and support. We also like to thank all the patients who joined the study.

Contributors Study concept and design: CAG and LYL; Acquisition of data: RSLL, KHL, CYY and SYC; Analysis and interpretation of data: CAG, LYL, GNC and $\mathrm{KKCH}$; Drafting of manuscript: $\mathrm{CAG}$ and LYL; Critical revision of the manuscript for important intellectual content: RSLL, KHL, CYY, SYC, GNC and KKCH; Statistical expertise: CAG, LYL and GNC; Acquisition of funding: CAG.

Funding This research was supported by a grant from Direct Grant for Research, The Chinese University of Hong Kong (Ref No. 4054249).

Competing interests None declared.

Patient consent for publication Obtained.
Ethics approval Approval was obtained from Institutional Review Board of the Chinese University of Hong Kong to conduct this prospective study (CREC 2015.624).

Provenance and peer review Not commissioned; externally peer reviewed.

Data sharing statement The datasets used and/or analysed during the current study are available from the corresponding author on reasonable request.

Open access This is an open access article distributed in accordance with the Creative Commons Attribution Non Commercial (CC BY-NC 4.0) license, which permits others to distribute, remix, adapt, build upon this work non-commercially, and license their derivative works on different terms, provided the original work is properly cited, appropriate credit is given, any changes made indicated, and the use is non-commercial. See: http://creativecommons.org/licenses/by-nc/4.0/.

\section{REFERENCES}

1. Bakker J, Nijsten MW, Jansen TC. Clinical use of lactate monitoring in critically ill patients. Ann Intensive Care 2013;3:12.

2. Fuller BM, Dellinger RP. Lactate as a hemodynamic marker in the critically ill. Curr Opin Crit Care 2012;18:267-72.

3. Browning R, Datta D, Gray AJ, et al. Peripheral venous and arterial lactate agreement in septic patients in the emergency department: a pilot study. Eur J Emerg Med 2014;21:139-41.

4. Contenti J, Corraze H, Lemoël F, et al. Effectiveness of arterial, venous, and capillary blood lactate as a sepsis triage tool in ED patients. Am J Emerg Med 2015;33:167-72.

5. Kelly AM. Can VBG analysis replace ABG analysis in emergency care?. Emerg Med J 2016;33:152-4.

6. Shapiro NI, Howell MD, Talmor D, et al. Serum lactate as a predictor of mortality in emergency department patients with infection. Ann Emerg Med 2005;45:524-8.

7. Lavery RF, Livingston DH, Tortella BJ, et al. The utility of venous lactate to triage injured patients in the trauma center. J Am Coll Surg 2000;190:656-64.

8. Ismail F, Mackay WG, Kerry A, et al. The accuracy and timeliness of a Point Of Care lactate measurement in patients with Sepsis. Scand J Trauma Resusc Emerg Med 2015;23:68.

9. Karon BS, Scott R, Burritt MF, et al. Comparison of lactate values between point-of-care and central laboratory analyzers. Am J Clin Pathol 2007;128:168-71.

10. Singer AJ, Taylor M, LeBlanc D, et al. ED bedside point-of-care lactate in patients with suspected sepsis is associated with reduced time to iv fluids and mortality. Am J Emerg Med 2014;32:1120-4.

11. Rossi AF, Khan D. Point of care testing: improving pediatric outcomes. Clin Biochem 2004;37:456-61.

12. Loveland ME, Carley SD, Cranfield N, et al. Assessment of the pain of blood-sugar testing: a randomised controlled trial. Lancet 1999:354:921-2.

13. Fauchère JC, Bauschatz AS, Arlettaz R, et al. Agreement between capillary and arterial lactate in the newborn. Acta Paediatr 2002;91:78-81.

14. Datta D, Grahamslaw J, Gray AJ, et al. Capillary and Venous Lactate Agreement: a pilot prospective observational study. Emerg Med J 2017;34:195-7.

15. Gaieski DF, Drumheller BC, Goyal M, et al. Accuracy of handheld point-of-care fingertip lactate measurement in the emergency department. West J Emerg Med 2013;14:58-62.

16. Bouzat $P$, Schilte $C$, Vinclair $M$, et al. Capillary lactate concentration on admission of normotensive trauma patients: a prospective study. Scand J Trauma Resusc Emerg Med 2016;24:82.

17. Manzon C, Barrot L, Besch G, et al. Capillary lactate as a tool for the triage nurse among patients with SIRS at emergency department presentation: a preliminary report. Ann Intensive Care 2015;5:7.

18. Bland JM, Altman DG. Statistical methods for assessing agreement between two methods of clinical measurement. Lancet 1986;1:307-10.

19. Wai AK, Chor CM, Lee AT, et al. Analysis of trends in emergency department attendances, hospital admissions and medical staffing in a Hong Kong university hospital: 5-year study. Int J Emerg Med 2009;2:141-8.

20. Altman DG. An introduction to medical statistics. 3rd ed. New York: Oxford University Press, 2000:272-5.

21. Frey B, Losa M. The value of capillary whole blood lactate for blood transfusion requirements in anaemia of prematurity. Intensive Care Med 2001:27:222-7.

22. Rathee K, Dhull V, Dhull R, et al. Biosensors based on electrochemical lactate detection: A comprehensive review. Biochem Biophys Rep 2016;5:35-54. 
23. Toffaletti J, Hammes ME, Gray R, et al. Lactate measured in diluted and undiluted whole blood and plasma: comparison of methods and effect of hematocrit. Clin Chem 1992;38:2430-4.

24. Ginsberg BH. Factors affecting blood glucose monitoring: sources of errors in measurement. J Diabetes Sci Technol 2009;3:903-13.
25. Pyne DB, Boston T, Martin DT, et al. Evaluation of the Lactate Pro blood lactate analyser. Eur J Appl Physiol 2000;82:112-6.

26. Tanner RK, Fuller KL, Ross ML. Evaluation of three portable blood lactate analysers: Lactate Pro, Lactate Scout and Lactate Plus. Eur J Appl Physiol 2010;109:551-9. 